Revue internationale P.M.E.

Économie et gestion de la petite et moyenne entreprise

\title{
Le marketing soutient-il l'avantage concurrentiel en petite entreprise?
}

\section{Marion Polge}

Volume 13, numéro 3-4, 2000

URI : https://id.erudit.org/iderudit/1008683ar

DOI : https://doi.org/10.7202/1008683ar

Aller au sommaire du numéro

\section{Éditeur(s)}

Presses de l’Université du Québec

ISSN

0776-5436 (imprimé)

1918-9699 (numérique)

Découvrir la revue

Citer cette note

Polge, M. (2000). Le marketing soutient-il l'avantage concurrentiel en petite entreprise? Revue internationale P.M.E., 13(3-4), 119-141.

https://doi.org/10.7202/1008683ar
Résumé de l'article

Alors que les grandes entreprises accordent une place croissante aux indicateurs fournis par les outils marketing pour assurer leur succès, cet article vise à montrer que le rôle de cette discipline dans la réussite de la petite entreprise concurrentielle peut être variable. Selon l'avantage concurrentiel recherché, la capacité organisa- tionnelle et l'environnement concurrentiel, le dirigeant peut accorder au marketing une place essentielle ou bien marginale dans sa stratégie.

Pour mieux comprendre comment s'établit puis évolue cette relation, une enquête qualitative longitudinale a été réalisée auprès de 10 petites entreprises du secteur agroalimentaire.

L'analyse évolutive montre que la relation entre marketing et avantage concurrentiel n'est pas figée. Cette enquête confirme que la petite entreprise peut réussir sans engager des moyens massifs dans une démarche marketing. 


\title{
Le marketing soutient-il l'avantage concurrentiel en petite entreprise?
}

Marion POLGE

Université d'Avignon

\section{MOTS CLÉS}

\author{
Avantage concurrentiel - Marketing - Petite entreprise \\ Compétence - Capacité - Capabilité - Clients - Dirigeant
}

\begin{abstract}
RÉSUMÉ
Alors que les grandes entreprises accordent une place croissante aux indicateurs fournis par les outils marketing pour assurer leur succès, cet article vise à montrer que le rôle de cette discipline dans la réussite de la petite entreprise concurrentielle peut être variable. Selon l'avantage concurrentiel recherché, la capacité organisationnelle et l'environnement concurrentiel, le dirigeant peut accorder au marketing une place essentielle ou bien marginale dans sa stratégie.

Pour mieux comprendre comment s'établit puis évolue cette relation, une enquête qualitative longitudinale a été réalisée auprès de 10 petites entreprises du secteur agroalimentaire.

L'analyse évolutive montre que la relation entre marketing et avantage concurrentiel n'est pas figée. Cette enquête confirme que la petite entreprise peut réussir sans engager des moyens massifs dans une démarche marketing.
\end{abstract}

\section{L'AUTEURE}

Marion Polge est maître de conférences en sciences de gestion à l'Université d'Avignon. Elle est membre de l'Équipe de recherche sur la firme et l'industrie (ERFI) à l'Université de Montpellier I. Adresse : Université d'Avignon et des Pays du Vaucluse, Case 20, 74, rue Louis-Pasteur, 84029 Avignon Cedex 1, France, tél. : 04901627 39, courriel : <marion.polge @univ-avignon.fr> 


\section{ABSTRACT}

While big companies give a growing place to the indicators provided by marketing tools in order to guarantee their success, the aim of this article is to show that the part of this discipline in the success of the small-sized competitive company could be variable. According to the competitive advantage requested, the organizational capacity and the competitive environment, the manager can give to marketing an essential or marginal place in his strategy.

In order to understand better the way this relation sets up and then evolves, a longitudinal qualitative survey has been carried out on ten small-sized companies of the food sector.

The evolutive analysis shows that the relation between marketing and competitive advantage is not frozen. This survey confirms that the small-sized company can succeed without investing substantial means in a marketing approach.

\section{RESUMEN}

Mientras que las grandes empresas conceden un puesto creciente a los indicadores proporcionados por los instrumentos de marketing para asegurar su éxito, este artículo muestra que el papel de esta disciplina en el triunfo de la pequeña empresa competidora puede ser variable. Según la ventaja competitiva deseada y buscada, la capacidad organizacional y la atmósfera competitiva, el director puede conceder al marketing una posición esencial o marginal en su estrategia.

Para comprender mejor como se establece y progresa esta relación, una encuesta de cualidad longitudinal fué realizada acerca de diez pequeñas empresas del sector agroalimentario.

El análisis evolutivo muestra que la relación marketing/ventaja competidora no está fijada. Esta encuesta confirma que la pequeña empresa puede lograr sin emplear medios importantes en un proyecto marketing.

\section{ZUSAMMENFASSUNG}

Im Gegensatz zu den Grossbetrieben, welche die Marketinginstrumente als entscheidend betrachten um den Unternehmenserfolg zu sichern, zeigt dieser Artikel, dass die Marketinginstrumente beim Erfolg von kleinen Unternehmen unterschiedlich zu beurteilen sind. Je nach Konkurrenzvorteil, der organisatorischen Kapazität und der Konkurrenzumwelt kann der Unternehmensleiter dem Marketing einen entscheidenden Platz oder eine Nebenrolle in seiner Strategie zuordnen.

Um die geschilderten Zusammenhänge besser zu verstehen, wurde eine qualitative Langzeituntersuchung bei zehn kleinen Nahrungsmittelbetrieben realisiert.

Die Analyse zeigt, dass die Beziehung Marketing/Konkurrenzvorteil nicht unumstösslich belegt werden kann. Die Untersuchung bestätigt, dass kleine Unternehmen Erfolg haben können ohne grosse Marketinginvestitionen. 


\section{Introduction}

Le rôle du marketing dans le maintien en compétitivité de l'entreprise est aujourd' hui reconnu, même si ce domaine reste souvent considéré en petite entreprise comme marginal, voire inexistant. Pourtant, Mendelssohn (1991), après avoir plaidé pour la mise en place de stratégie marketing quelle que soit la taille de l'entreprise, soutient que «les entreprises les plus petites qui vont prospérer ne peuvent tout simplement pas se permettre d'ignorer le concept marketing ».

Cette affirmation peut être présentée différemment. Lorsque Mendelssohn parle d' « ignorance », il fait implicitement référence à un concept clairement défini. Or, les petites comme les grandes entreprises sont confrontées quotidiennement à des marchés de plus en plus concurrentiels et évolutifs. Mais pour survivre et maintenir leur compétitivité, les dirigeants de petites entreprises mettent en place des stratégies de différenciation afin de sensibiliser, puis de fidéliser leur clientèle. Par le choix de leurs partenaires, ils essaient de donner à leur environnement une configuration évitant l'exposition à une concurrence directe. Le contexte dans lequel ils mènent une réflexion marketing n'est pas semblable à celui des grandes entreprises nationales ou internationales qui évoluent dans un environnement plus vaste et plus ouvert à la concurrence. Dans le cadre de la petite entreprise, le concept marketing, tel qu'il est généralement défini, risque d'être dénaturé ; plutôt que d' «ignorance », il serait bon de parler de définition spécifique pour la petite entreprise.

Aux problèmes de définition du concept marketing s'ajoutent des questions d'objectifs et d'ambition distinguant petite et grande entreprise. L'avance obtenue par une petite entreprise sur les concurrents ne s'exprime pas nécessairement en part de marché, et un avantage concurrentiel n'exige pas une position de leader. Le dirigeant de petite entreprise qui mène une réflexion marketing dans ces conditions ne cherche pas une victoire sur des concurrents qu'il a parfois du mal à définir, mais seulement la fidélisation de la clientèle.

L'objet de cet article est de montrer que, dans le cadre de la petite entreprise, le marketing n'a pas toujours un rôle fondamental dans l'aboutissement de l'avantage concurrentiel. La définition donnée au marketing par le dirigeant de petite entreprise peut être à l'origine de cette situation.

La présentation du cadre conceptuel comporte deux parties. Dans un premier temps, la spécificité de l'avantage concurrentiel en petite entreprise est étudiée, ensuite, le marketing sera examiné et mis en relation avec l'avantage concurrentiel. Cela permettra de construire une grille d'analyse et de formuler des propositions de recherche quant à la nature de la relation entre marketing et avantage concurrentiel.

Afin de mieux comprendre comment le dirigeant d'une petite entreprise définit le marketing et le valorise dans sa stratégie concurrentielle, une étude 
qualitative auprès d'une dizaine de petites entreprises (moins de 15 salariés) a été réalisée. Les résultats obtenus permettent de concevoir une typologie d'entreprises et d'identifier les différents cheminements que suivent les dirigeants.

\section{Rôle du marketing dans l'avantage concurrentiel en petite entreprise : cadre conceptuel}

L'avantage concurrentiel et le marketing vont être présentés de manière générale, puis dans le champ spécifique de la petite entreprise.

\subsection{Essai de définition de l'avantage concurrentiel en petite entreprise}

L'avantage concurrentiel a été défini par les éléments qui le soutiennent dans l'entreprise et ceux qui accréditent son apparition dans l'environnement. Les spécificités organisationnelles et environnementales qui caractérisent la petite entreprise justifient une adaptation conceptuelle de la notion d'avantage concurrentiel.

\subsubsection{Débats autour du concept d’avantage concurrentiel}

Durant la dernière décennie, les définitions de l'avantage concurrentiel ont été abondantes ; Porter est à l'origine des avancées théoriques et pragmatiques majeures. La définition qu'il a proposée en 1985 semble aujourd'hui admise : " une firme acquiert un avantage concurrentiel en exerçant des activités stratégiquement importantes, à meilleur marché ou mieux que ses concurrents ».

S'il est admis que l'avantage concurrentiel permet d'améliorer la performance ${ }^{1}$, le véritable débat se trouve dans ses stratégies d'acquisition. Deux principaux courants de pensée coexistent. Le premier, apparu au début des années 1980, peut être qualifié d' « approche environnementale » ou encore d' " approche écologique » ; Porter en a été l'un des principaux défenseurs. Dans cette perspective, l'avantage concurrentiel s'acquiert sur le long terme, grâce à une planification stratégique établie dans le respect d'un certain déterminisme environnemental (South, 1981; Gerstein et Reisman, 1982 ; Portnoff, 1990). L'avantage concurrentiel doit alors permettre une victoire durable sur la concurrence, qui se déroule en toute transparence

1. La performance est un concept polysémique difficile à appréhender. Il se décline aussi bien en analyse des résultats obtenus ex post (Bouquin, 1986), qu'en processus, c'est-àdire en « mise en actes d'une compétence qui n'est qu'une potentialité » (Baird, 1986, dans Bessire, 1999). Elle est la traduction de la détention d'un avantage concurrentiel. 
et dans des conditions d'imitabilité et d'échange parfait des ressources ${ }^{2}$ (Lawles, 1991 ; Fulmer et Goodwin, 1988). Tout d'abord accueillis avec enthousiasme, les modèles prescriptifs proposés ont ensuite été vivement critiqués, notamment pour leur négligence de la spécificité de chaque entreprise (Aharoni, 1993; Mahoney et Pandian, 1992). D'ailleurs, Porter (1999) réfute lui-même cette analyse au profit d'approches plus contingentes.

C'est par rapport à ces critiques que s'est développée à la fin des années 1980 une nouvelle approche des questions relatives à l'analyse concurrentielle, qualifiée d'approche «resource-based»: chaque entreprise emprunte un itinéraire unique pour accéder à l'avantage concurrentiel. Ce parcours consiste à transformer les ressources $^{3}$ (tangibles ou non) en compétences distinctives (Wernerfelt, 1984; Conner, 1991 ; Barney, 1991). Les compétences distinctives et les actifs stratégiques composent la capacité que l'entreprise cherche à insérer dans son environnement (Grant, 1991), c'est-à-dire la «capabilité » (capability); (Hall, 1993; Bartmess et Cerny, 1993 ; Lenz, 1980). L'avantage concurrentiel est conditionné par l'existence d'un «fit» entre la capabilité de l'entreprise et son environnement. L'entreprise participe à la construction de l'environnement et n'en subit pas systématiquement une pression unilatérale.

Ces deux courants de pensée, s'ils offrent une certaine complémentarité dans l'étude de l'avantage concurrentiel, laissent cependant des zones d'ombre qui prennent de l'ampleur dans le cadre de la petite entreprise.

\subsubsection{Avantage concurrentiel en petite entreprise: proposition d'une nouvelle approche du concept}

Il est aujourd'hui commun d'évoquer le rôle prédominant que joue le dirigeant dans la gestion de la petite et même de la moyenne entreprise (d'Amboise et Muldowney, 1988 ; Marchesnay, 1992 ; Dianich et Gupta, 1993). L'abondance des travaux sur l'entrepreneuriat témoigne du grand nombre d'interrogations le concernant. Il en est de même pour les questions de concurrence, puisque plusieurs travaux empiriques (Bennett, 1983 ; Covin et Covin, 1990) ont mis en évidence que les petites entreprises

2. La «chaîne de valeur », instrument conçu par Porter, permet de repérer pour chaque entreprise la création de valeur suivant les différentes activités et de formuler la stratégie à adopter. Bien que reconnue pour sa pertinence, elle préconise un cloisonnement des activités de l'entreprise, qui est parfois délicat à réaliser concrètement. L'entreprise développe ensuite une stratégie générique (de différenciation ou de coûts bas). De plus, elle n'aborde pas les problèmes de lien avec l'environnement. Pour cela, il est nécessaire d'utiliser le modèle des « cinq forces de la concurrence».

3. Dans cette approche, les ressources sont inimitables et non échangeables. 
réussissent en réagissant avec agressivité face à un environnement hostile. Quelle que soit la situation, le comportement du dirigeant est présenté comme contraint, « forcé» (Sauers, 1983) par l'oppression concurrentielle.

Le problème peut être appréhendé différemment: la transparence environnementale telle qu'elle est définie par l' « approche écologique » des populations voudrait que l'environnement s'impose à tous les acteurs qui le composent. Mais il est possible de définir le niveau concurrentiel de l'environnement par le comportement de chaque acteur. Chaque dirigeant bâtit son « micro-environnement » par le choix de ses interlocuteurs et souligne ou tempère le rôle de certaines variables (Chappoz, 1991). Ce phénomène prend de l'ampleur lorsque la taille de l'entreprise régresse, car le dirigeant a tendance à s'isoler pour négocier seulement avec ses partenaires les plus proches et les plus sensibles à la capacité développée dans l'entreprise.

Par sa perception, le dirigeant définit l'environnement (concurrentiel et non concurrentiel), il choisit la capacité développée par l'entreprise et le «fit» qui déterminera son adéquation avec l'environnement (Polge, 1996). Ses choix de valorisation des ressources et des compétences de l'entreprise sont motivés par ses buts (personnels et professionnels). Ainsi, les buts et la perception du dirigeant sont à l'origine de la nature et de la puissance de l'avantage concurrentiel, exprimée en termes de performance ${ }^{4}$ (Day et Nedungadi, 1996). Le schéma 1 présente l'ensemble de ces relations.

Il ne s'agit pas d'une victoire irréversible sur la concurrence (Porter, 1985), mais d'un moyen de perdurer en consolidant la cohésion stratégique (Porter, 1999) telle que la perçoit le dirigeant. Dans ces conditions, qu'en est-il de la notion de marketing?

\subsection{Marketing et succès concurrentiel en petite entreprise}

Comme l'avantage concurrentiel, le concept marketing peut difficilement conserver sa définition intrinsèque en petite entreprise. Certains éléments peuvent devenir discutables alors que le concept est défini par le dirigeant qui en presse l'utilité.

4. La nature de l'avantage concurrentiel est caractérisée par les composants du différentiel de capacité. La puissance est liée aux critères de performance retenus pour l'évaluation de l'avantage concurrentiel. On distingue la performance financière (taux de profit, rentabilité, etc.), la performance concurrentielle (fidélisation, satisfaction des clients, part de marché) et la performance organisationnelle (climat organisationnel, cohésion administrative, culture, etc.). 


\section{SCHÉMA 1}

\section{L'avantage concurrentiel en petite entreprise}

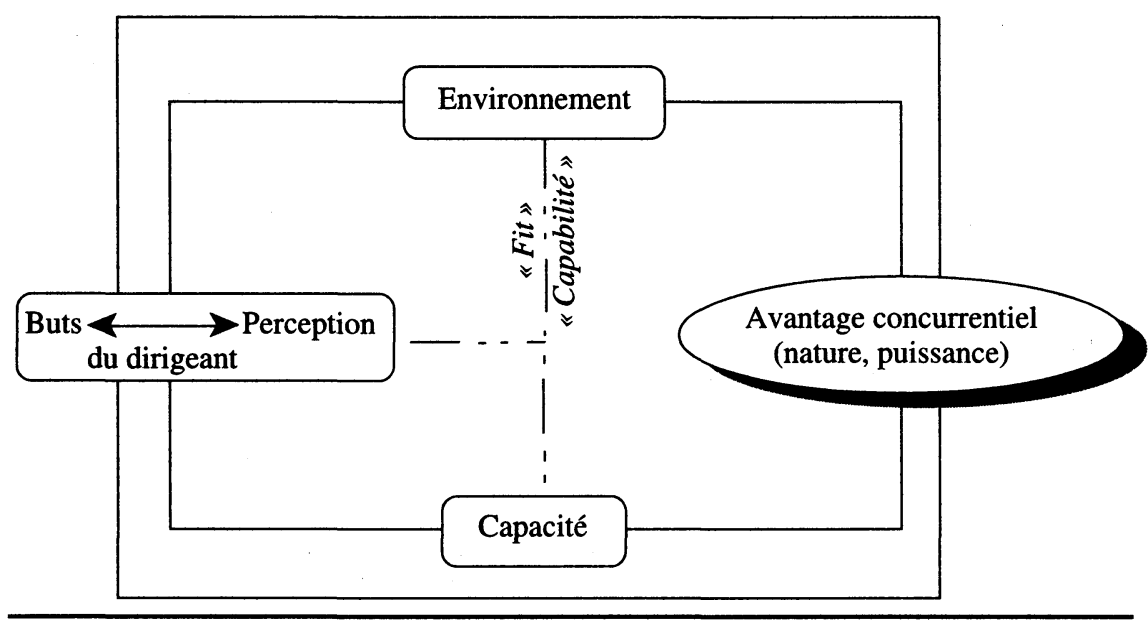

\subsubsection{Définition atypique du marketing}

D'après Webster (1992), l' approche du marketing a beaucoup évolué et « la recherche marketing a gagné une place prédominante dans la pratique du management comme un moyen d'aligner les capacités / aptitudes productives de l'entreprise aux besoins du marché ». Alors qu'initialement le concept marketing était réduit à un rassemblement des objectifs autour de la réalisation de profits et de ventes maximales (Felton, 1959; Ackoff, 1970), celles-ci sont devenues les conséquences de l'objectif de satisfaction de la clientèle.

Disposant d'outils de plus en plus élaborés (bâtis à partir de méthodes quantitatives), le marketing est devenu une fonction centrale de l'entreprise (Houston, 1986). Les plus grandes ont été amenées à départementaliser la fonction marketing (vente, distribution, conception, etc.), tandis que les petites entreprises ont conservé une activité centralisée autour du dirigeant, parfois secondé d'un conseiller ou d'un commercial (Webster, 1992). La prédominance de la fonction marketing dans les choix stratégiques de l'entreprise (Day et Wensley, 1983 ; Conrath, 1993) a progressivement écarté la petite entreprise dont le dirigeant ne peut ou ne veut pas suivre ce schéma structurel ${ }^{5}$. Cela pourrait expliquer que certaines études (Taylor, 1993)

5. La notion de marché est souvent jugée comme obscure par le dirigeant qui lui préfère celle de client, beaucoup plus proche des réalités quotidiennes (Pacitto et Tordjman, 1998). 
tendent aujourd'hui à faire croire que les dirigeants de petites entreprises ne s'attardent pas sur la fonction ou le comportement marketing et sont en conséquence limités dans le management (Mayo, 1991). Pourtant, ils cherchent à identifier les consommateurs potentiels, à fidéliser la clientèle en satisfaisant les besoins et parfois à l'étendre (Boag et Dastmalchian, 1988), même lorsque leur démarche reste très informelle (Herrmann, 1998). Carson et Cromie (1989) parlent de «style marketing distinctif ».

Certes, le faible montant des ressources ne permet pas à ces dirigeants d'amplifier le rôle du marketing comme c'est le cas pour les grandes entreprises (Davis, Hills et Laforge, 1985 ; Weinrauch et al., 1991). Mais la question qui importe touche plutôt l'impact du marketing sur la stratégie, l'avantage concurrentiel et la performance de la petite entreprise (Carson, 1985).

\subsubsection{Impact du marketing sur la performance de la petite entreprise}

La relation entre marketing et avantage concurrentiel devient problématique en petite entreprise sur le plan qualitatif et quantitatif.

Le marketing peut avoir une importance très variable dans le différentiel de capacité (Pacitto et Tordjman, 1998). Le dirigeant peut conduire une réflexion marketing ponctuelle ou continue (Meziou, 1989). Il peut ne retenir qu'une définition partielle du marketing (stratégique, opérationnel, relationnel, etc.) ou bien la modifier selon le problème posé (Ford et Rowley, 1979; Shama, 1993). Il impliquera le marketing comme une source majeure ou mineure d'avantage concurrentiel, selon sa perception de l'organisation, de la capacité qui s'en dégage et de l'avantage concurrentiel qu'il souhaite acquérir pour être performant. C'est en quelque sorte la relation bi-univoque entre la capacité de l'entreprise et le microenvironnement que construit le dirigeant qui conditionne le rôle du marketing dans la démarche stratégique. Si l'environnement contribue de manière déterminante à l'apparition de la capacité / aptitude, le dirigeant adopte alors une démarche marketing telle qu'elle est décrite dans les travaux de portée générale. En revanche, si la capacité de l'entreprise guide la construction d'un environnement seulement propice à sa traduction en capabilité puis en avantage concurrentiel, il sera possible d'envisager l'existence d'un marketing d'une autre nature.

Deux propositions peuvent être formulées :

1. Le dirigeant a tendance à définir le marketing comme une démarche nécessaire pour communiquer sa capacité au marché.

Reprenant les principes de définition de l'avantage concurrentiel en petite entreprise (présentés dans le schéma 1), le schéma 2 illustre la situation suggérée par cette proposition. Le dirigeant puise la source de l'avantage concurrentiel dans les compétences de l'organisation (le plus souvent liées à un savoir-faire ou à un 


\section{SCHÉMA 2}

Le marketing comme un moyen de commercialisation de la capacité de l'entreprise

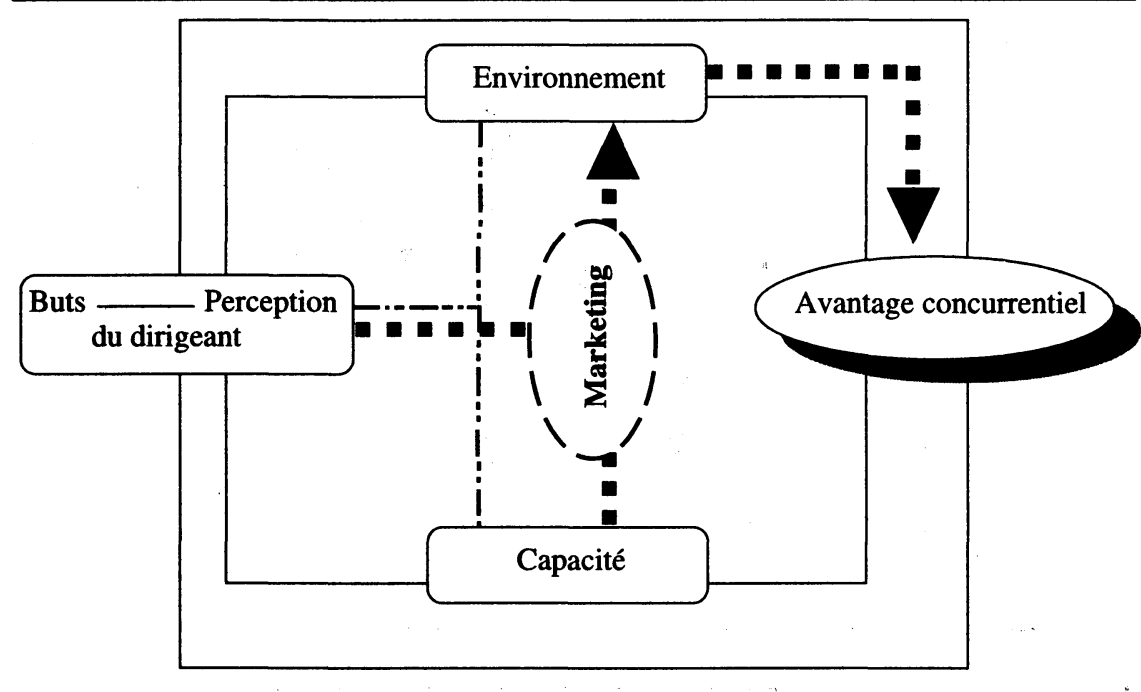

métier) et cherche ensuite à l'insérer dans l'environnement. Le fit pourrait émaner de la capacité de l'entreprise qui se révèle toutefois insuffisante pour qu'apparaisse un véritable avantage concurrentiel. Le dirigeant utilise ainsi le marketing pour soutenir l'insertion environnementale, alors que la capacité de l'entreprise est déjà établie. Dans ce cas, le marketing s'apparente plus à de l'action commerciale et s'écarte de sa définition initiale.

2. Réciproquement, le dirigeant peut relever dans l'environnement une attente insatisfaite ou bien créer un besoin. Il puise la source de l'avantage concurrentiel dans un constat environnemental et bâtit ensuite la capacité de son organisation (voir schéma 1). Le schéma 3 reproduit cette logique en y intégrant le marketing. La réflexion marketing, d'une part, précède la caractérisation de la capacité (flèche 1 sur le schéma 3) et, d'autre part, accompagne sa commercialisation (flèche 2 sur le schéma 3). Le dirigeant pense que le marketing soutient directement la stratégie de l'entreprise et son avantage concurrentiel, comme le montre le schéma 3.

Au cours du temps, un même dirigeant peut modifier ses buts et sa définition du marketing. Il peut bénéficier de contacts extérieurs, recruter un spécialiste, changer de rôle dans l'entreprise, etc., et reconsidérer l'ensemble de sa stratégie. Ces deux propositions ne sont donc pas exclusives et laissent entendre au contraire que l'influence du marketing dans la performance peut revêtir plusieurs aspects selon le moment considéré. 
SCHÉMA 3

Le marketing comme source principale d'avantage concurrentiel

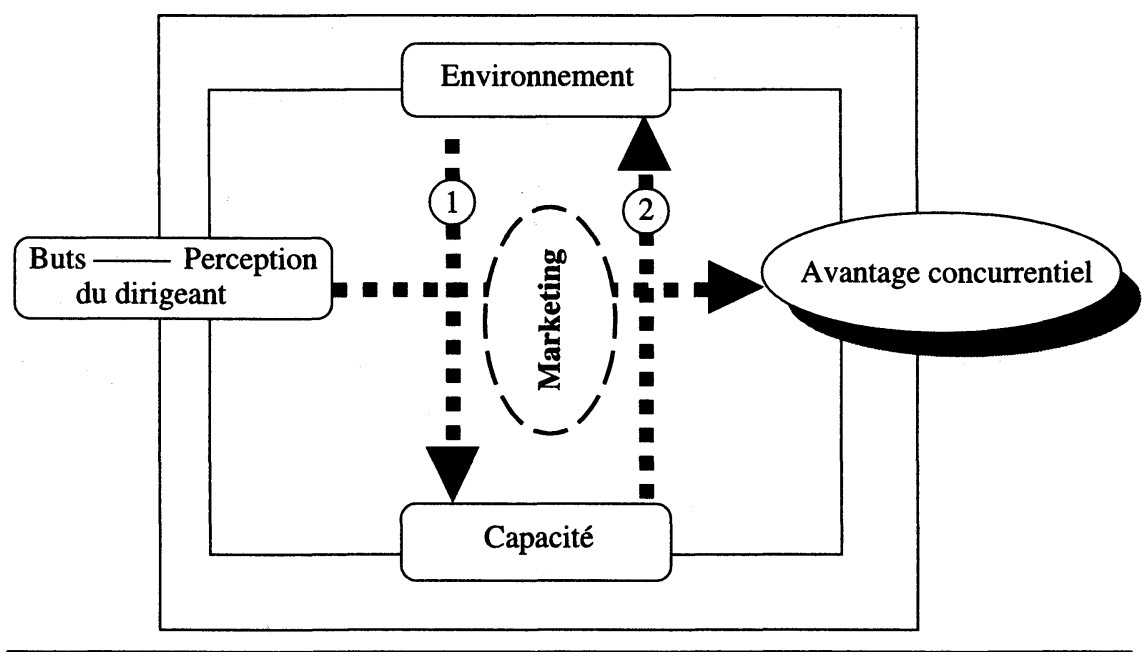

\section{Nécessité d'une méthodologie qualitative}

Le choix d'une méthodologie qualitative va être argumenté ainsi que l'outil qui a permis de mettre en lumière les résultats.

La méthodologie se justifie par le caractère exploratoire de la recherche et par le positionnement de la problématique. Ces deux points vont être respectivement abordés afin de mieux comprendre l'outil utilisé.

\subsection{Nature de la recherche et positionnement du problème}

Ce travail a pour objectif de montrer l'existence d'une relation entre la perception du marketing par le dirigeant et la caractérisation de l'avantage concurrentiel de son entreprise. Il vise à mieux comprendre l'importance du marketing dans les entreprises concurrentielles, ce qui ne nécessite pas, dans un premier temps, d'en mesurer l'intensité.

Tel qu'il est posé, le problème se situe dans le cadre des méthodes qualitatives pour deux raisons principales. D'une part, ce sont des explications plus que des descriptions de phénomènes qui sont recherchées dans ce travail (Wacheux, 1996). Lorsque la réponse n'est contrainte ni dans la forme, ni dans le fond (Das, 1983), l'enquêté peut librement exprimer sa perception des données ${ }^{6}$ et offrir

6. Les données obtenues sont hautement subjectives. 
des informations inattendues (Behling, 1980; Morgan et Smircich, 1980). D'autre part, les méthodes qualitatives permettent d'étendre l'explication à des données passées. Or, ce travail s'inscrit dans la dynamique. L'enquêté peut expliquer comment il perçoit le processus d'évolution et de construction de l'entreprise (Harrigan, 1983; Weick, 1989).

L'état d'avancement empirique de cette de recherche, mais surtout la nature du problème qu'il pose et les objectifs poursuivis justifient le choix de la méthode d'enquête qualitative.

\subsection{Présentation de l'outil utilisé}

L'étude de cas permet de travailler en profondeur une unité ou un petit nombre d'unités d'analyse; la puissance explicative est plus liée à la profondeur qu'à l'étendue de l'étude. La question portant ici sur la compréhension de l' "essence » d'un phénomène (Wacheux, 1996), cette technique semble adaptée puisqu'elle permet de comprendre "pourquoi les décisions sont prises, comment elles sont exécutées et avec quels résultats» (Yin, 1989; Moati, 1992).

L'enquête a été réalisée auprès de 10 petites entreprises (moins de 15 salariés) du secteur agroalimentaire en Languedoc-Roussillon ${ }^{7}$. Chaque dirigeant a été rencontré au cours de plusieurs entretiens semi-dirigés pendant une période de deux ans. Ce travail portant sur les données perçues par le dirigeant (unique décideur stratégique), il a été le seul membre de l'entreprise interrogé. Les informations recueillies (concernant les buts, les caractéristiques du dirigeant, l'environnement, l'organisation, l'avantage concurrentiel, la définition du marketing et le style de gestion) ont été rassemblées dans une grille $d$ 'indicateurs homogènes afin de réduire les risques de biais et d'établir des comparaisons de données. Les variables retenues ont été déclinées à partir de la grille d'analyse présentée en première partie (schéma 1) et retenant, pour variables principales, les buts du dirigeant, l'avantage concurrentiel et la performance.

Les buts du dirigeant ont été appréhendés à partir de la typologie de Marchesnay (1992) qui retient la pérennité, la croissance de l'entreprise, l'indépendance financière et l'autonomie de prise décision comme guides des choix stratégiques. L'avantage concurrentiel peut revêtir une configuration différente en fonction de

7. Les entreprises devaient répondre à certains critères de sélection :

- autonomie de prise de décision par le dirigeant ;

- indépendance juridique ;

- création antérieure à cinq ans ;

- dirigeant en place depuis plus de deux ans;

- activité de transformation et non de production agricole. 
l'environnement (concurrentiel et non concurrentiel), mais aussi de la capacité de l'entreprise (voir les parties supérieure et inférieure du schéma 1). Ces deux axes indissociables pour caractériser l'avantage concurrentiel ont été examinés distinctement puis conjointement afin de faire apparaître clairement la source fondamentale de la réussite. L'analyse de l'environnement s'est appuyée sur les travaux de $\operatorname{Porter}^{8}(1982,1985,1997)$, tandis que la capacité de l'entreprise a été appréciée selon les apports de Barney (1991), Hall (1993) et surtout Wernerfelt (1984).

Enfin, la raison d'être de l'avantage concurrentiel qu'est l'amélioration de la performance a été décomposée en trois indicateurs principaux. La performance organisationnelle, que Powell (1992) décline selon le climat organisationnel, la culture organisationnelle et la compétence administrative. La performance concurrentielle (Hansen et Wernerfelt, 1989 ; Fiol, 1991) est également composée de trois paramètres : la part de marché (Gilbert et Strebel, 1991), les niches profitables et les coûts bas (Zahra et Das, 1993) et la satisfaction et la fidélisation de la clientèle (Day et Wensley, 1988). Bien que faisant intégralement partie de la caractérisation de la performance concurrentielle, la part de marché n'a pas été retenue comme indicateur pertinent. Chakravarthy (1986) souligne ses limites pour certaines entreprises. C'est le cas de la petite entreprise du secteur agroalimentaire qui ne fournit qu'une part infime de la production nationale, malgré une activité dynamique. La performance économique, qui connaît depuis quelques années de profondes mutations (Lorino, 1991), permet d'apprécier les profits supérieurs à la normale engendrés par l'avantage concurrentiel (Williams et Smart, 1993; Zahra et Das, 1993). Cet indicateur apparait intimement lié aux deux précédents. Mais le sens de cette relation pourrait se révéler intéressant quant à la nature de l'avantage recherché et aux moyens mis en œuvre.

\section{Résultats de la recherche}

Les récits obtenus ont permis de relever trois types de discours ainsi que leurs processus d'évolution.

\subsection{Essai de typologie d'entreprises}

Tout d'abord, il n'apparaît pas véritablement de différence dans l'implication commerciale des dirigeants rencontrés, selon la source d'avantage concurrentiel $q u$ 'ils perçoivent. Tous les groupes d'entreprises rassemblent indifféremment des dirigeants qui délèguent ou assument l'activité commerciale, montrent une certaine agressivité ou se sentent au contraire vulnérables. En revanche, le terme « marketing »

8. La démarche a principalement consisté à identifier les cinq forces de la concurrence et la nature des relations. 
est quasiment absent de leur vocabulaire, même lorsque la réflexion marketing est sous-jacente dans leur discours. Les notions rattachées à cette discipline et jugées importantes par le dirigeant ne sont pas toujours les mêmes.

Le schéma 4 montre les trois principaux groupes d'entreprises. Reprenant les variables présentées dans les schémas 1,2 et 3 , il visualise les relations dominantes établies à l'issue de l'enquête. Plusieurs enseignements sont à souligner concernant les trois variables principales retenues.

Parmi les quatre buts du dirigeant qui ont été examinés, seule «l'autonomie de prise de décision » au sens de Marchesnay (1992) est apparue indifféremment dans toutes les entreprises. Les dirigeants interrogés ont semblé peu frileux à l'égard de l'endettement, dans la mesure où celui-ci n'altérait pas « la discrétionnarité des décisions, l'autonomie de gestion » (Marchesnay, 1992). Ce but ne figure donc pas dans le schéma 4 ; la pérennité, la croissance ${ }^{9}$ et l'indépendance financière ont participé, avec l'avantage concurrentiel et la performance à établir une distinction entre trois groupes d'entreprises différents.

Les entretiens ont permis de constituer plusieurs formes de caractérisation de l'avantage concurrentiel. Dans le discours des dirigeants, il est apparu une distinction nette entre, d'une part, la capacité de se démarquer de la concurrence grâce à la qualité du produit et, d'autre part, l'impossibilité de développer un avantage concurrentiel à partir du seul produit. Dans le second cas, le dirigeant a été conduit à concevoir un ensemble de prestations pour pouvoir se maintenir sur le marché ${ }^{10}$. Compte tenu de la difficulté à repérer la source décisive de cet avantage concurrentiel, il a été caractérisé à partir du différentiel complexe qui le soutient.

Tous les dirigeants sont apparus sensibles à la notion de performance économique qu'ils estiment, à juste titre, incontournable pour assurer la pérennité de leur entreprise. Ils se sont montrés peu disposés à dévoiler leur taux de rentabilité ou leur retour sur investissements. Les données quantitatives que nous avons pu recueillir en matière économique sont donc relativement pauvres. En revanche, les discours interprétatifs pléthoriques ont facilité une analyse fouillée des liens pouvant exister entre les deux autres indicateurs de performance. Certains dirigeants ont privilégié la performance concurrentielle et d'autres, la performance organisationnelle pour améliorer leurs résultats économiques. En centrant l'analyse sur ces deux indicateurs, nous avons pu mettre en évidence un troisième type de comportement

9. Toutefois, seule la croissance de la clientèle est apparue souhaitable. Même si l'on peut supposer que celle-ci implique une extension d'activité et donc, une croissance de l'entreprise, les dirigeants n'ont pas explicitement manifesté le souhait de faire crô̂tre leur entreprise.

10. Il peut s'agir de divers services, de partenariat, de sous-traitance, liés à une renonciation d'identité... 
SCHÉMA 4

Relations entre l'avantage concurrentiel et le marketing en petite entreprise

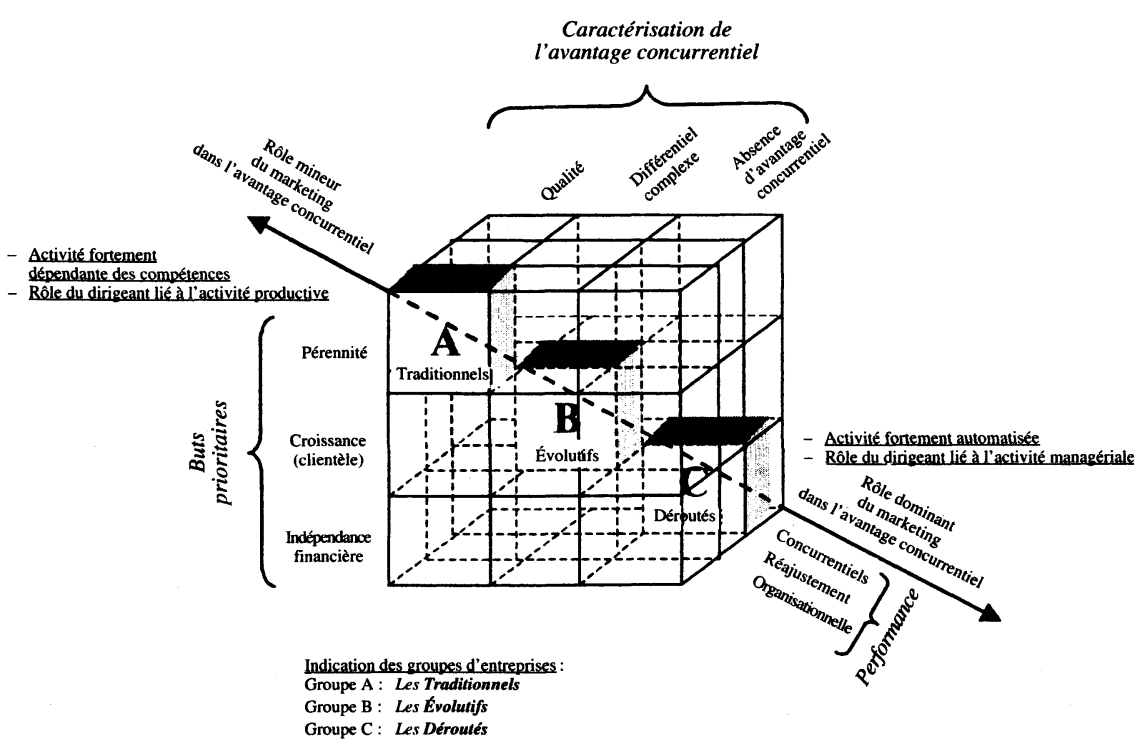

plus nuancé (schéma 4) : certains dirigeants effectuent un réajustement en fonction des problèmes à traiter, de l'importance des enjeux concurrentiels ou encore de la nécessité de mobilisation de leur équipe.

Les groupes A et B rassemblent respectivement quatre entreprises et deux entreprises seulement figurent dans le groupe $\mathbf{C}$.

Dans les entreprises du groupe $\mathrm{A}$, le dirigeant pense que la qualité intrinsèque du produit soutient principalement l'avance sur les concurrents. Acquis au fil du temps, le savoir-faire permet d'établir une différenciation par rapport aux concurrents (petits ou grands) et de se protéger des offensives extérieures. La préservation et l'enrichissement lent d'un métier traditionnel justifient la caractérisation des entreprises de ce groupe comme celui des «traditionnels». Les dirigeants de ces entreprises perçoivent la qualité comme une source d'avantage concurrentiel néanmoins insuffisante et lui associent une stratégie commerciale qu'ils assument ou délèguent partiellement. Ils ne dissocient pas l'avantage concurrentiel de la capacité de l'entreprise et pensent que la performance organisationnelle est le meilleur moyen pour maintenir la compétitivité de leur entreprise. La durée de vie de leur entreprise est un but dominant qui semble dicter les choix stratégiques de ces dirigeants. 
Pour les dirigeants des entreprises traditionnelles, le marketing est réduit à la satisfaction d'une clientèle sensible au savoir-faire développé par l'entreprise ; ils ne cherchent pas à connaître l'appréciation qu'ont les clients de leurs produits. Convaincus de la supériorité de leur niveau de qualité, ils utilisent leur capacité comme un acquis et repèrent ensuite leurs partenaires environnementaux. Souvent embryonnaires, les techniques commerciales employées se traduisent par une présence très forte sur le terrain. Que ce soit géographiquement et / ou en utilisant un canal de distribution différent, ces entreprises évitent l'affrontement concurrentiel, car le dirigeant redoute un manque de flexibilité opérationnelle. La définition du marketing se rapproche ici de celle de l'action commerciale, au détriment d'une véritable réflexion marketing.

Dans le groupe $\mathrm{B}$, les dirigeants ne distinguent pas une source dominante d'avantage concurrentiel. La qualité de leur produit ne les épargne pas de la concurrence, mais c'est un ensemble complexe de compétences et d'actifs (tangibles ou non) qui leur assure une clientèle régulière. Bien qu'ils maîtrisent un savoir-faire, ils ne le jugent pas suffisant pour maintenir une avance concurrentielle. Fortement automatisée, leur activité peut être implantée sans difficulté dans les autres entreprises. Ils recherchent de nouveaux clients de manière permanente par crainte de ne pas pouvoir réagir rapidement à une offensive concurrentielle. Ces dirigeants sont attentifs aux critères de performance concurrentielle, mais ne négligent pas systématiquement la performance organisationnelle : ils procèdent à un réajustement entre les deux critères de performances.

Ils définissent le marketing comme un moyen de repérer l'orientation à donner à la capacité de l'entreprise. Exerçant une activité facilement imitable et peu différenciée, ces dirigeants sont à l'écoute du marché afin d'y saisir les attentes des consommateurs et d'adapter leur organisation. La réflexion marketing soutient la capabilité de l'entreprise et permet de créer le fit entreprise-environnement. Les membres de ce groupe sont qualifiés d' «évolutifs » du fait de l'adaptation permanente de la capacité / aptitude face aux changements environnementaux.

Une seule entreprise enquêtée se trouve dans une phase transitoire à la suite d'un changement de dirigeant il y a deux ans et demi. Appartenant initialement aux «traditionnels », elle rejoint les «évolutifs », car le nouveau dirigeant estime que la qualité ne suffira pas pour se maintenir sur le marché de façon durable.

Les deux entreprises du groupe $\mathrm{C}$ n'ont pas réussi à développer un différentiel de capacité qui les protège de la concurrence. Leur situation extrêmement critique nous incite à les qualifier de « déroutées ». L'une dispose d'un savoir-faire semblable à celui des grandes entreprises de sa branche d'activité et n'a communiqué à ses clients aucun critère de différenciation crédible et la seconde a subi la pression d'une clientèle qui n'accordait aucune importance à la différenciation qualité perçue par le dirigeant. Les deux dirigeants ont refusé l'endettement pour préserver leur 
indépendance financière. Sur un plan stratégique, ils ont combiné les démarches des dirigeants « traditionnels » et «évolutifs ».

Dans ces deux entreprises, une capacité a été développée sans que soit menée une réflexion marketing. Le premier dirigeant ne s'est pas interrogé sur le niveau de satisfaction des consommateurs ni sur les débouchés de son produit; il s'est appuyé sur une intime conviction, plus que sur une véritable analyse. Très automatisée, l'activité n'exige pas un savoir-faire particulier, ce qui explique que le dirigeant ne s'intéresse que très modérément à la performance organisationnelle. Il s'inquiète constamment des raisons de la perte de ses clients et ne comprend pas que la capacité de l'entreprise ne crée pas un critère de différenciation.

Le second dirigeant s'est limité à l'approvisionnement de son plus gros client et a laissé s'installer la dépendance. Responsable d'une entreprise familiale, il n'a pas souhaité négliger totalement la performance organisationnelle, mais il a été plus attentif à la performance concurrentielle à travers la fidélisation de son plus gros client, sans comprendre ses critères de satisfaction, ni quel était son pouvoir de négociation.

\subsection{Processus d'évolution de la relation entre marketing et avantage concurrentiel}

Les entreprises « traditionnelles » étudiées sont toutes familiales et ont parfois été créées par leur actuel dirigeant. L'activité est exercée à partir d'un savoir-faire et l'automatisation est limitée. Le processus d'acquisition de l'avantage concurrentiel se fait par une adaptation de la configuration environnementale à la capacité de l'entreprise. La rigidité opérationnelle et stratégique n'est brisée que sous contrainte (institutionnelle ou concurrentielle); le changement organisationnel délibéré est conséquent à une réflexion interne.

Les dirigeants rencontrés connaissent la logique suivie par les «évolutifs», mais ne l'approuvent pas; ils perçoivent l'identité organisationnelle acquise sur le long terme comme une force concurrentielle et un atout commercial. D'ailleurs, leur rôle dans l'entreprise reste au fil du temps lié à l'activité productive alors qu'ils délèguent une partie de l'exploitation et de la gestion.

Il y a une dizaine d'années, trois entreprises «évolutives » appartenaient au groupe des «traditionnelles». Les dirigeants perçoivent leur situation actuelle comme la conséquence de l'évolution environnementale qui a accéléré l'obsolescence de leur savoir-faire. Ils attribuent leur succès à la transformation de la contrainte en atout : ils ont assimilé certaines données exogènes pour construire une nouvelle capacité. Les choix stratégiques sont précédés systématiquement d'une réflexion sur les données environnementales et les variables marketing. Toutefois, 
les dirigeants pensent que ce processus fragilise leur entreprise ; ils souhaiteraient rejoindre les « traditionnels », mais convaincus de l'impossibilité de cette éventualité, ils s'adaptent continuellement au nouveau processus. Paradoxalement, ils perçoivent leur situation simultanément comme une contrainte et un atout. Parallèlement à cette évolution, le rôle du dirigeant dans l'entreprise a changé. Auparavant proche de l'activité productive, il s'en éloigne progressivement pour se concentrer sur la gestion administrative et commerciale où se situe le cœur de l'avantage concurrentiel. La plupart des dirigeants du groupe des « évolutifs » n'ont pas créé leur entreprise ; ils ont succédé à leurs parents ou ont racheté une entreprise familiale. Certains donnent cette explication pour justifier leur implication réduite ou inexistante dans l'activité productive et leur intérêt pour la gestion stratégique, marketing et commerciale.

S'agissant des deux entreprises « déroutées », l'une a déjà été « traditionnelle » et l'autre, « évolutive ». L'entreprise antérieurement « traditionnelle» a été évincée par les contraintes extérieures dont l'influence a été mal gérée. Le dirigeant s'efforce de redevenir « traditionnel » en rebâtissant sa capacité et en modifiant son insertion environnementale. L'entreprise issue du groupe des «évolutifs » est en situation d'échec définitif, puisqu'elle est en vente; son dirigeant estime le retour impossible.

En résumé, les cas étudiés laissent penser que la relation entre marketing et avantage concurrentiel peut suivre deux évolutions :

1. Le dirigeant cherche à maintenir la stabilité organisationnelle et s'y implique directement ; il minimise le rôle du marketing dans la stratégie. Le concept marketing est alors défini comme un moyen de repérer la clientèle et l'avantage concurrentiel puise ses fondements dans une capacité organisationnelle bâtie sur un métier. Le marketing est quasiment assimilé à la gestion commerciale. Ces entreprises sont le plus souvent familiales et cherchent à durer plutôt qu'à affronter la concurrence.

2. Le dirigeant est contraint de subir un changement de capacité. S'il l'accepte, le marketing devient dominant dans l'avantage concurrentiel; s'il le refuse, l'entreprise peut échouer. Le marketing est défini comme une source d'information (marketing informationnel et relationnel) permettant de mener une réflexion sur les choix stratégiques (marketing stratégique) et de valoriser la capacité ainsi développée (marketing opérationnel). Lorsque la source d'avantage concurrentiel devient diffuse, le dirigeant s'appuie sur les outils du marketing pour effectuer ses choix stratégiques. Soucieux de la survie de son entreprise, il est animé par un besoin continu et sécurisant d'extension de clientèle. Cette finalité justifie son rôle central dans le management. 


\subsection{Discussion et conclusion}

\subsubsection{Concept marketing en petite entreprise}

Les travaux concernant le marketing en petite entreprise aboutissent à des conclusions abusivement pessimistes (McDaniel et Parasuraman, 1986; Kinsey, 1987). En fait, les outils et les concepts rattachés au marketing qui peuvent trouver leur pertinence auprès de grandes entreprises nationales ou internationales sont souvent dénaturés en petite entreprise (Brown, 1982 ; Hills, 1985), ce qui explique leur faible utilisation; le vocabulaire des dirigeants en témoigne. Ils ne parlent pas de «marchés » mais de «clients», ils redoutent le terme de «concurrent» et lui préfèrent celui de «confrère » (ce qui est conforme aux résultats de Marchesnay, 1988). La relation entreprise-marché se réduit à une relation entre dirigeant et partenaires choisis de manière réticulaire. L'enquête qui vient d'être présentée montre que le concept de marketing est pourtant très présent en petite entreprise ; il est même inhérent, puisque l'insertion environnementale est renforcée. De ce fait, les résultats montrant que le marketing est absent de la petite entreprise ont peutêtre seulement le tort d'utiliser globalement un concept sans l'adapter aux besoins et au cadre théorique. Le rôle prédominant du dirigeant le rend responsable des relations environnementales. Dans ces conditions, il n'utilise pour sa réflexion et sa démarche que certains aspects du marketing. Le concept marketing semble se décliner en plusieurs définitions complémentaires plus ou moins vastes. Le dirigeant les retient selon le problème posé et le moment considéré, sans que ces définitions soient nécessairement généralisables à l'ensemble des entreprises.

\subsubsection{Marketing et compétitivité}

L'enquête a mis en évidence que le concept marketing est plus ou moins présent dans l'acquisition d'un avantage concurrentiel selon les définitions données par le dirigeant. Il ne soutient pas l'avantage concurrentiel entièrement, mais sa contribution peut être très marquée. Pour les entreprises « traditionnelles », l'insertion est conséquente au développement de la capacité de l'entreprise. La fonction marketing consiste seulement à identifier des consommateurs qui pourraient être satisfaits par les produits et/ou services proposés.

Les dirigeants des entreprises «évolutives » s'appuient sur le marketing pour créer leur capacité, puis pour la véhiculer. Le concept marketing est très présent dans l'acquisition de l'avantage concurrentiel. Il en est l'initiateur et l'insertion environnementale de la capacité s'effectue ensuite selon les bases établies à partir de la réflexion marketing. Plus flexibles (sur le plan stratégique et opérationnel) que les entreprises «traditionnelles», ces dernières sont également plus fragiles. C'est pourquoi le dirigeant est simultanément attentif à plusieurs critères de performance. Les entreprises « déroutées » sont les plus problématiques; elles montrent 
l'incohérence du raisonnement des dirigeants qui interprètent les informations pouvant définir leur positionnement. Pour ce type d'entreprise, une aide à l'établissement du diagnostic stratégique pourrait éviter l'enlisement définitif.

\section{Conclusion}

Ces résultats ne permettent pas de conclure sur le fait que le marketing peut être la source unique d'avantage concurrentiel ; au contraire, les deux concepts apparaissent indissociables, mais leur lien n'est pas exclusif. Ils montrent surtout que la clé de voûte du succès se situe dans la cohérence entre la stratégie globale et la stratégie marketing et non dans l'utilisation intensive, continue et exacerbée des outils marketing. La relation entre le marketing et l'avantage concurrentiel est apparue extrêmement plurielle et contingente.

Il aurait été intéressant, pour consolider les résultats obtenus, d'examiner des petites entreprises appartenant à d'autres secteurs d'activité. Il est en effet possible que l'agroalimentaire présente des spécificités sur ce point précis.

Une enquête empirique paraît également incontournable pour confirmer les résultats de ce travail exploratoire.

\section{Bibliographie}

ACKOFF, R.L. (1970), A Concept of Corporate Planning, New York, Wiley.

AHARONI, Y. (1993), «In search of the unique: can firm specific advantages be evaluated ?», Strategic Management Journal, vol. 30, $\mathrm{n}^{\circ}$ 1, janvier, p. 31-49.

BAIRD, L. (1986), Managing Performance, New York, John Wiley.

BARNEY, J.B. (1991), «Firms resources and sustained competitive advantage », Journal of Management, vol. 17, $\mathrm{n}^{\circ} 1$, mars, p. 99-120.

BARTMESS, A. et K. CERNY (1993), « Building competitive advantage through a global network of capabilities », California Management Review, vol. 35, $\mathrm{n}^{\circ}$ 2, hiver, p. 78-103.

BEHLING, O. (1980), « The case for the natural science model for research in organizational behavior and organization theory », Academy of Management Review, vol. $5 \mathrm{n}^{\circ} 4$, p. 483-490.

BENNET, D. (1983), «Small firms and the law of competition on the EEC », European Small Business Journal, vol. 1, $\mathrm{n}^{\circ}$ 2, p. 25-37.

BESSIRE, D. (1999), «Définir la performance», Comptabilité-Contrôle-Audit , t. 5, vol. 2, p. $127-150$.

BoAG, D.A. et A. DASTMALChIAN (1988), «Market vulnerability and the design and management of the marketing function in small firms », Journal of Small Business Management, vol. 26, $\mathrm{n}^{\circ}$ 4, octobre, p. 37-42. 
BouQuin, H. (1986), Le contrôle de gestion, Paris, Presses universitaires de France.

BROWN, R. (1982), «Introducing marketing into the smaller firm », Journal of European Industrial Training, vol. 6, $\mathrm{n}^{\circ} 3$, p. 25-28.

CARSON, D.J. (1985), « The evolution of marketing in small firms », European Journal of Marketing, vol. 19, no 5, p. 7-17.

CARSON, D. et S. CROMIE (1989), « Marketing planning in small enterprises : A model and some empirical evidence », Journal of Marketing Management, vol. 5, p. 33-49.

CHAKRAVARTHY, B.S. (1986), « Measuring strategic performance », Strategic Management Journal, vol. 7, p. 437-458.

CHAPPOZ, Y. (1991), «La gestion de l'interactivité entreprise / environnement », Revue internationale PME, vol. 4, no 3, p. 53-75.

COLLERAN, J.F. (1985), « Strategic marketing considerations for the small firm », European Journal of Marketing, vol. 19, $\mathrm{n}^{\circ}$ 5, p. 17-31.

CONNER, K.R. (1991), «A historical comparison of resource-based theory and five schools of thought within industrial organization economics : do we have a new theory of the firm? ", Journal of Management, vol. 17, $\mathrm{n}^{\circ} 1$, mars.

CONRATH, D.W. (1993), «The marketplace : concepts and issues », Journal of Small Business and Entrepreneurship, vol. 10, $\mathrm{n}^{\circ}$ 4, juillet-septembre, p. 69-80.

Covin, J.G. et T.J. Covin (1990), « Competitive aggressiveness, environmental context, and small firm performance », Entrepreneurship Theory and Practice, vol. 14, $\mathrm{n}^{\circ} 4$, été, p. 35-50.

D'Amboise, G. et M. MULDowney (1988), « Management theory of small business : attempts and requirements », Academy of Management Review, vol. 13, no 2, p. 226240.

DAS, H. (1983), « Qualitative research in organizational behaviour », Journal of Management Studies, vol. 3, no 20, p. 301-314.

DAVIS, C.D., G.E. HILLS et R.W. LAFORGE (1985), « The marketing/ small enterprise paradox : a research agenda », International Small Business Journal, vol. 3, $\mathrm{n}^{\circ} 3$, p. 31-42.

DAY, G.S. et P. NEDUNGADI (1996), « Managerial representations of competitive advantage », Journal of Marketing, vol. 58, p. 31-44.

DAY, G.S. et R. WENSLEY (1983), « Marketing theory with a strategic orientation », Journal of Marketing, vol. 47, automne, p. 79-89.

DAY, G.S. et R. WENSLEY (1988), « Assessing advantage : a framework for diagnosing competitive superiority », Journal of Marketing, vol. 52, avril, p. 1-20.

DIANICH, D.F. et J.N.D. GUPTA (1983), « Decision analysis for small business », American Journal of Small Business, vol. 8, $\mathrm{n}^{\circ}$ 2, décembre, p. 15-56.

Felton, A.P. (1959), « Making the marketing concept work », Harvard Business Review, vol. 37, juillet-août, p. 55-65.

FIOL, C.M. (1991), « Managing culture as a competitive resource : an identity-based view of sustainable competitive advantage », Journal of Management, vol. 19, $\mathrm{n}^{\circ} 1$, p. 191-211. 
FORD, D et T.P. ROWLEY (1979), « Marketing and the small industrial firm », Management Decision, vol. 17, $\mathrm{n}^{\circ} 2$, p. 144-156.

FULMER, W.E. et J. GOODWIN (1988), « Differenciation begins with the consumer », Business Horizons, vol. 31, $\mathrm{n}^{\circ}$ 5, septembre-octobre.

GERSTEIN, M. et H. REISMAN (1982), «Creating competitive advantage with computer technology », Journal of Business Strategy, vol. 3, no 1, été, p. 53-60.

Gilbert, X. et P. STREbel (1991), «Developping competitive advantage», dans H. Mintzberg, The Strategy Process, Concepts, Context, Cases, $2^{\mathrm{e}}$ édition, Englewood Cliffs, N.J., Prentice Hall.

GRANT, R.M. (1991), «Porter's competitive advantage of the nation an assessment », Strategic Management Review, vol. 12, p. 535-548.

HALL, R. (1993), « A framework linking intangible resources and capabilities to sustainable competitive advantage », Strategic Management Journal, vol. 14, p. 607-618.

HANSEN, G.S. et B. WERNERFELT (1989), « Determinants of firm performance : the relative importance of economic and organizational factors », Strategic Management Journal, vol. 10, p. 399-411.

HARRIGAN, K.R. (1983), « Research methodologies for contingency approaches to business strategy », Academy of Management Review, vol. 8 n 3, p. 398-405.

HERMANN, J.L. (1998), «Le marketing en PME manufacturière : les pratiques de quelques entreprises lorraines lors du développement de produit », Présentation au Congrès international francophone de la PME, Nancy-Metz.

HILLS, G.E. (1985), «Market analysis in the business plan : venture capitalists' perceptions », Journal of Small Business Management, p. 38-46, janvier.

Houston, F.S. (1986), «The marketing concept : what it is and what it is not ", Journal of Marketing, vol. 50, avril, p. 81-87.

JONES, T.C. et G.R. SEILER (1988), « The rapidly growing pump company : marketing for competitive advantage », Planning Review, vol. 16, no 3, mai, p. 30-48.

KINSEY, J. (1987), «Marketing and the small manufacturing firm in Scotland : findings of a pilot survey », Journal of Small Business Management, vol. 25, $\mathrm{n}^{\circ} 2$, avril, p. 18-25.

LAWLES, M.W. (1991), « Commodity bundling for competitive advantage : strategic implications », Journal of Management Studies, vol. 28, mai.

LENZ, R.T. (1980), «Strategic capability : a concept and framework for analysis », Academy of Management Review, vol. 5, $\mathrm{n}^{\circ} 2$, p. 225-234.

LORINO, P. (1991), Le contrôle de gestion stratégique, Paris, Éditions Dunod, 213 p.

MCDANiel, S. et A. PARAsuraman (1986), «Practical guidelines for small business marketing research », Journal of Small Business Management, vol. 24, $\mathrm{n}^{\circ} 1$, janvier, p. 1-9.

MAHONEY, J.T. et R. PANDIAN (1992), « The resource-based view within the conversation of strategic management », Strategic Management Journal, vol. 13, p. 363-380.

MARCHESNAY, M. (1988), «La mercatique en petite entreprise», Revue internationale $P M E$, vol. $1, \mathrm{n}^{\text {os }}$ 3-4. 
MARCheSnAy, M. (1992), «La PME : une gestion spécifique », Problèmes économiques, $\mathrm{n}^{\circ} 2$ 276, mai, p. 26-32.

MAYO, M.A. (1991), « Ethical problems encountered by U.S. small businesses in international marketing », Journal of Small Business Management, avril, p. 51-59.

MENDELSSOHN, J. (1991), «Small firms and the marketing mission », Accountancy, $\mathrm{n}^{\circ} 107$, janvier, p. 96-97.

Meziou, F. (1989), « Areas of strength and weakness in the adoption of the marketing concept by small manufacturing firms », Journal of Small Business Management, vol. $29, \mathrm{n}^{\circ} 4$.

MoATI, P. (1992), «Méthodologie d'analyse de secteur : pour une reformulation évolutionniste du "paradigme S-C-P" ", Document de recherche du CREDOC, Paris I.

MORGAN, G. et L. SMIRCICH (1980), « The qualitative research», Academy of Management Review, vol. 5, n 4, p. 491-500.

PACITTO, J.C. et F. TORDJMAN (1998), «Peut-on parler de marketing dans la TPE?», Présentation au Congrès international francophone de la PME, Nancy-Metz.

POLGE, M. (1996), L'avantage concurrentiel en petite entreprise, thèse de doctorat Nouveau Régime, Université de Montpellier I, janvier.

PORTER, M.E. (1982), Choix stratégiques et concurrence, Paris, Economica, 426 p.

PORTER, M.E. (1985), L'avantage concurrentiel, comment devancer ses concurrents et maintenir son avance, Paris, InterÉditions, $647 \mathrm{p}$.

PORTER, M.E. (1999), «Qu'est-ce que la stratégie ?», dans La concurrence selon Porter, Paris, Éditions Village Mondial, 475 p.

PORTNOFF, A.Y. (1990), «Produire sur mesure : le client roi », Futuribles, novembre, p. 67-73.

POWELL, C.P. (1992), « Research notes and communications strategic planning as competitive advantage », Strategic Management Journal, vol. 13, p. 551-558.

SAUERS, D.G. (1983), «Using statistics to improve the competitive position of a small business », American Journal of Small Business, vol. 8, $\mathrm{n}^{\circ} 1$, juillet-septembre, p. $52-65$.

SHAMA, A. (1993), "Marketing strategies during recession : a comparison of small and large firms », Journal of Small Business Management, vol. 31, $\mathrm{n}^{\circ} 3$, juillet, p. 62-72.

SOUTH, S.E. (1981), « Competitive advantage : the cornerstone of strategic thinking », The Journal of Business Strategy, vol. 1, $\mathrm{n}^{\circ}$ 4, printemps, p. 15-25.

TAYLOR, D.B. (1993), "Marketing: contrasting attitudes of small and large manufacturers », Journal of Small Business and Entrepreneurship, vol. 10, $\mathrm{n}^{\circ}$ 3, avril-juin, p. 116-120.

WACHEUX, F. (1995), "L'utilisation de l'étude de cas dans l'analyse des situations de travail », Actes du VI $I^{e}$ Congrès de l'AGRH, Poitiers, édition dirigée par P. Louart, p. 127-135.

WACHEUX, F. (1996), Méthodes qualitatives et recherche en gestion, Paris, Economica, $290 \mathrm{p}$. 
WEBSTER, F.E. (1992), « The changing role of marketing in the corporation », Journal of Marketing, vol. 56, octobre, p. 1-17.

WEICK, K.E. (1989), «Theory construction as disciplined imagination », Academy of Management Review, vol. 14, $\mathrm{n}^{\circ}$ 5, p. 516-531.

WeINRAUCH, J.D., O.K. MANN, P.A. RoBinson, et J. PHARR (1991), « Dealing with limited financial resources : a marketing challenge for small business », Journal of Small Business Management, vol. 29, $\mathrm{n}^{\mathrm{O}}$ 4, octobre, p. 44-54.

WENSLEY, R. et G.S. DAY (1983), « Marketing theory with a strategic orientation », Journal of Marketing, vol. 47, automne, p. 79-89.

WERNERFELT, B. (1984), «A resource-based view of the firm», Strategic Management Journal, vol. $5, \mathrm{n}^{\circ} 2$, p. 171-180.

WiLliams, A. MC et D.L. SMART (1993), «Efficiency v. structure-conduct-performance : implications for strategy research and practive », Journal of Management, vol. 19, $\mathrm{n}^{\mathrm{0}} 1$, p. 63-78.

YIN, R.K. (1989), Case Study Research, $2^{\mathrm{e}}$ édition, Londres, Sage publications.

ZAHRA, S.A. et S.R. DAS (1993), «Building competitive advantage on manufacturing resources », Long Range Planning, vol. 6, $\mathrm{n}^{\circ}$ 2, p. 90-100. 